\title{
Confirmation of the stubborn-error effect in human multiple-choice verbal learning
}

\author{
MELVIN H. MARX and KATHLEEN MARX \\ University of Missouri, Columbia, Missouri 65211
}

\begin{abstract}
Data from a previously reported experiment (Marx \& Marx, 1980) were analyzed to replicate an earlier result relating to error repetition (Marx, 1971; Marx, Witter, \& Farbry, 1973). A total of 112 high school students from five psychology classes were given a multiplechoice verbal learning task in their regular classroom setting. For the group as a whole, the more often a particular error had been repeated on prior successive trials, the more likely it was to recur. However, only 21 (about one-fifth) of the subjects showed this stubbornerror effect, and male students were primarily responsible for it (13 of 42 males showed the effect, compared with only 8 of 70 females).
\end{abstract}

Data previously reported in a larger study (Marx \& Marx, 1980) were examined to extend the analysis of a phenomenon reported earlier (Marx, 1971; Marx, Witter, \& Farbry, 1973). In those reports, it was shown that the probability of making some particular error in the multiple-choice learning situation increases regularly with the number of prior successive repetitions of that error (Marx, 1971; Marx, Witter, \& Mueller, 1972). It was our intention to assess the generality of the phenomenon of especially stubborn errors.

\section{METHOD}

Only the training data were used from the previously reported experiment (Marx \& Marx, 1980; Day 2 data). A multiplechoice verbal learning task was administered to 112 high school psychology students in their normal classroom setting. The students selected one of four alternatives for each of 32 cue words. All of the alternatives completed a commonly used phrase (e.g., "room," "rank," "mate," and "ring" to the cue "class"). The students were told that they would need to guess on the first trial because correct alternatives had been randomly determined, but that they should attempt to remember and repeat on subsequent trials as many of the correct responses as possible. All cues and alternatives were presented by slide projector, and correct responses were provided after all of the subjects had responded. Variations in task procedure over the five classes are described in the previous report (Marx \& Marx, 1980).

\section{RESULTS}

Figure 1 shows the probability of error repetition as a function of the number of prior successive occurrences of the same erroneous response to the cue. For all the subjects, this probability obviously increased, up to $100 \%$ after six successive occurrences. ${ }^{1}$

Supported in part by a Research Career Award from the National Institute of Mental Health and a grant from the Army Research Institute for Behavioral and Social Sciences, both to the first author. The opinions expressed herein are those of the authors and do not constitute endorsement by the U.S. Army.
In order to assess the extent to which such error persistence was generally expressed throughout the subject sampling, a subgroup of the most frequent repeaters was formed. All subjects who had repeated at least three errors on the second trial and had carried a high proportion of repetitions of the same errors for at least two additional trials were included (except for the subjects in one class, who had only three trials, because of apparatus breakdown, so that repetitions on just one additional trial were required).

The 21 subjects thus selected for persistent error repetitions gave successive repetition percentages of $29.2 \%(104 / 356), 56.7 \%$ (59/104), 60.0\% (21/35), $85.0 \%(17 / 20), 53.3 \%(7 / 13)$, and $100 \%(5 / 5)$. (The numerical variations in numerator-to-next-denominator relationships are due to the different stopping points in the various classes.)

The remaining subjects, selected on this arbitrary ad hoc basis for their relatively low error-repetition rates, gave corresponding successive percentages of

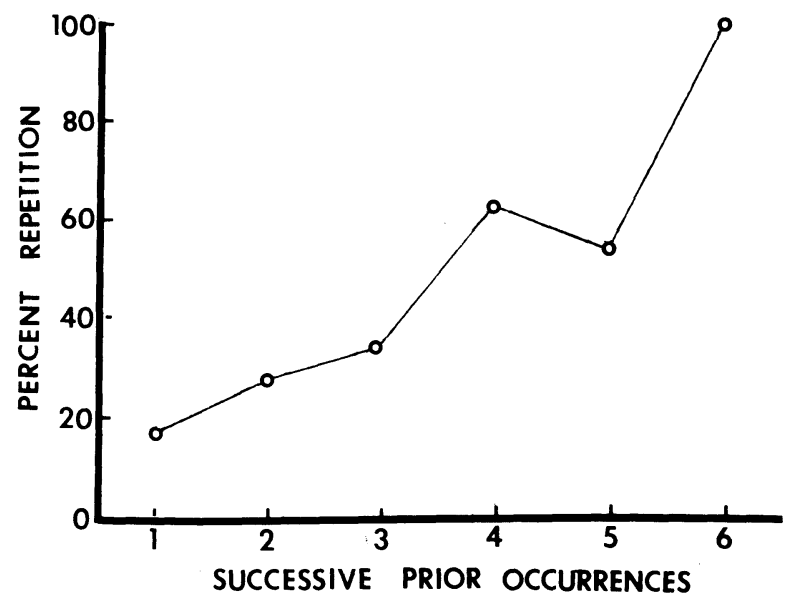

Figure 1. Percent repetition of first-trial errors as a function of the number of prior successive occurrences. 
$14.8 \%(289 / 1,948), 18.7 \%(53 / 285), 17.6 \%(6 / 34)$, and $0 \%(0 / 6)$. It is clear that the effect shown in Figure 1 was due to the small proportion of subjects, approximately one-sixth of the total group, who provided the most frequent repetitions of specific errors.

Selection of these 21 high repeaters was done without knowledge of the sex of the subjects. When sex of subject was subsequently checked, a substantial proportion of the male subjects (13 of 42) were found to be high repeaters, whereas a much smaller proportion of the female subjects ( 8 of 70 ) were so categorized. This difference in proportions was evaluated by a chi-square test and found to be statistically reliable $\left[\chi^{2}(1)=6.56, p<\right.$ .05 (6.63 required for $p=.01)]$.

The overall error-repetition curves for the separate classes are shown in Figure 2. An increasing percentage of error repetitions as a function of prior successive occurrences clearly occurred for all of the classes except the fifth, and this exception reflected the very small number (one) of high-frequency repeaters in that class.

\section{DISCUSSION}

When measured in groups, the phenomenon of incremental error strength seems to be well established. The present results not only confirm those previously reported (Marx, 1971; Marx et al., 1972) but also suggest the dependence of the incremental effect upon a small number of individuals in each group. When too small a number of such individuals is present, the effect is not demonstrated (cf. Figure 2, Class 5, with only one high repeater).

Class 5 learned with delayed feedback (correct answers for all of the items provided at the end of each trial). However, supposition that this factor was responsible for the failure of Class 5 to show the effect is contradicted by the fact that Class 4, which did show the usual effect, also learned with delayed feedback. A more suggestive difference between these two classes was the writing out of the words by Class 5 , compared with the circling of an identifying number by Class 4 . Perhaps the greater attention presumably required in writing the words helped to break error strings. The fact that female students were reliably less often categorized as high-frequency repeaters is consistent with this suggestion, in light of their

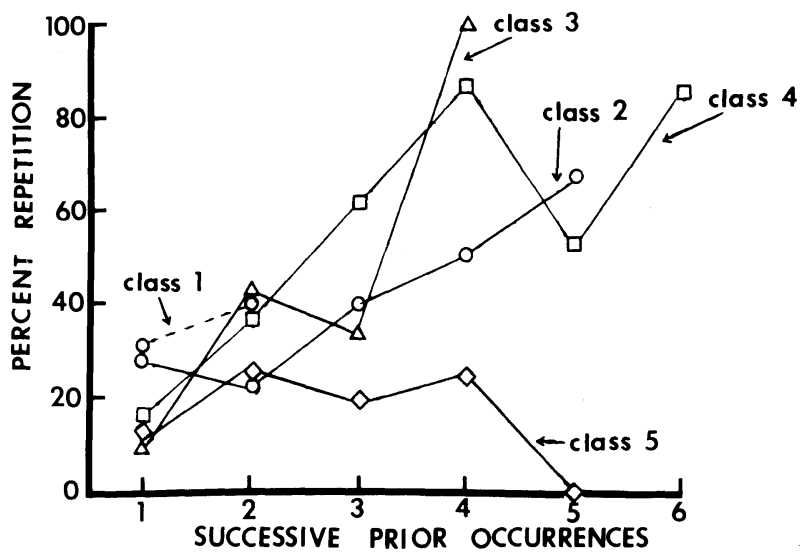

Figure 2. Percent repetition of first-trial errors as a function of successive prior occurrences for the five classes. commonly observed superiority in verbal performance, relative to males (Maccoby \& Jacklin, 1974).

The suggestion is also supported by the recent demonstration of a generalized inhibition of learning in males, but not females, as a function of being required to score, as well as observed paired performers' responses (Marx, 1979). The surprisingly high concentration of persistent error repeaters among males in this study also may be related to their tendency to repeat both errors and correct responses more often than females when they select the responses (i.e., serve as performers) rather than merely observing them (Marx et al., 1973).

Gender differences aside, the implications of the apparent dependence of the stubborn-error effect on certain individuals are not entirely clear. Several questions are raised. For example, is the phenomenon restricted to such a small proportion of individuals, for some individual-differences reasons, or would more individuals show it if given greater opportunity to do so? Also, how much consistency will high repeaters show over successive tasks and across a variety of tasks?

A logical next step would be to obtain large amounts of data over and across tasks on some subjects in order to determine more precisely the conditions under which certain errors do become stronger with continued occurrence and the individual differences related to this effect. The empirical difficulties that one can reasonably expect to encounter in such an undertaking are so formidable as to discourage research along these lines. Nevertheless, the fact that such incremental error tendencies do occur, even if under special conditions and not generally, seems to us to be of sufficient potential importance to justify paying continued theoretical and experimental attention to the problem.

Finally, we might briefly suggest the role of the present error analysis in the more general setting of learning theory. For example, what is the relationship of the kind of error string that we have measured to the errors that Norman (Note 1) has recently examined? Our assumption is that the genesis of stubborn errors of the sort treated in this report is qualitatively different from the "slips" described by Norman, attributed by him to processing accidents. The slips discussed by Norman may be more intrinsically interesting, but the stubborn errors that we have analyzed are probably more common and, in some ways, perhaps represent a more central problem in learning and performance because they are more likely to be generated during the normal course of behavior. For this reason, they also seem to be different from the ubiquitous role accorded errors in Harlow's (1959) error-set theory. In any event, all of these types of error analysis seem to us to be important-certainly more important than the relatively slight amount of experimental and theoretical attention they have thus far received would suggest-and their further experimental examination is indicated.

\section{REFERENCE NOTE}

1. Norman, D. A. Slips of the mind and an outline for a theory of action (Tech. Rep. 88). San Diego: University of California, Center for Human Information Processing, November 1979.

\section{REFERENCES}

HaRLOW, H. F. Learning set and error factor theory. In S. Koch (Ed.), Psychology: A study of a science (Vol. 2). New York: McGraw-Hill, 1959.

Maccoвy, E. E., \& Jacklin, C. N. The psychology of sex differences. Stanford, Calif: Stanford University Press, 1974.

MARX, M. H. Increased probability of error repetition as a function of number of successive prior repetitions. Perceptual and Motor Skills, 1971, 32, 544-546.

MarX, M. H. Multiple-choice learning of line-drawn facial 
features: II. Sex differences. Bulletin of the Psychonomic Society, 1979, 14, 439-441.

MARX, M. H., \& MARX, K. Repetition of errors in learning and memory as a function of their prior associative strength. Bulletin of the Psychonomic Society, 1980, 16, 435-438.

Marx, M. H., Witter, D. W., \& Farbry, J. Greater repetition of errors under performance compared to observation in multiplechoice human learning. Perceptual and Motor Skills, 1973, 37, 949-950.

Marx, M. H., Witter, D. W., \& Mueller, J. H. Additional data on probability of error repetition following varying numbers of successive prior repetitions. Perceptual and Motor Skills, 1972, 34, 525-526.

\section{NOTE}

1. One subject who repeated an inordinate number of errors (a total of 16 repetitions through five trials) was discarded from the data analysis on the grounds that these data did not represent a reasonably representative learning effort.

(Received for publication November 24, 1980.) 